\title{
Dielectric function of a colloidal solution near an interface with pure solvent
}

\author{
R. Stepanyan* \\ Physics of Complex Fluids Laboratory, Applied Physics Deptartment, University of Twente, P.O. Box 217, 7500 AE Enschede, \\ The Netherlands
}

(Received 24 November 2003; revised manuscript received 31 March 2004; published 7 October 2004)

\begin{abstract}
The effective dielectric function of colloidal solution in the vicinity of a solution-pure solvent interface is discussed. A simple expression is derived for the transverse and longitudinal components of the dielectric permittivity tensor $\overleftrightarrow{\epsilon}$ as a function of the distance from the interface. In the approximation made, the sole effect responsible for the $\overleftrightarrow{\epsilon}$ anisotropy and space dependence is the asymmetry in the pair correlation function. Consequently, the final result is valid for dilute solutions only. The effect of polydispersity of the solute particles is considered in an approximate manner as well.
\end{abstract}

DOI: 10.1103/PhysRevB.70.132201

Being one of the most promising types of materials, composites have continued to attract a lot of attention during several decades. This interest is caused, first of all, by a unique opportunity to combine sometimes nearly opposite properties in one material, thus allowing a very wide range of applications. In addition to their commercial and industrial importance, composites pose many theoretically challenging questions. The problem of the effective dielectric function of an inhomogeneous medium is one such. Its history starts from classical works ${ }^{1-3}$ and continues nowadays with new methods being proposed. ${ }^{4-9}$ The aim of these studies is generally the same: to obtain the effective permittivity as a function of the concentration, size, and shape of the inclusions. The researchers are strongly motivated by a possible experimental application, because the analysis of the composite dielectric spectra allows one to derive information on the microstructure or on the properties of the dispersed component. ${ }^{10,11}$ To go beyond the simple Maxwell-Garnett ${ }^{2}$ approximation, which contains no information about polydispersity, etc., one has to take into account the interparticle interactions as well. This makes the analytical solution of the problem quite complicated ${ }^{7,12}$ and stimulates the employment of numerical techniques. ${ }^{13}$

Although the main effort has been made toward the investigation of three-dimensional systems, the properties of inhomogeneous media in two-dimensional (2D) confinement conditions also received some attention. ${ }^{14,15}$ In the bulk system the interaction of an inclusion particle with the external field and the other inclusions describes the situation completely. The presence of a surface or an interface makes interactions with the electrostatic images caused by this interface equally important. The polarizability of a single particle on a substrate has been addressed in a number of works ${ }^{16}$ covering not only spherical particles but also truncated spheres and spheroids. This allowed one to calculate the surface dielectric susceptibilities of polarizable entities arranged in a 2D square lattice. For disordered 2D systems it has been shown that the spatial arrangement in the neighborhood of the test particle is very important. ${ }^{15}$

Related problems appear in the physics of colloids and suspensions if one considers the effective dielectric function near a liquid-liquid phase boundary. ${ }^{17}$ The susceptibility of the inhomogeneous phase can be averaged over a small volume (but large enough to include many particles) situated at
PACS number(s): 77.55.+f, 77.22.Ch, 77.84.Lf, 78.20.Ci

the given distance $z(z>0)$ from the interface. Therefore the dielectric permittivity tensor becomes spatially dependent and can be represented as a function of $z$ in the case of a flat interface: $\overleftrightarrow{\epsilon}(z)$. In theoretical works the interaction between inclusions and their images has been claimed to be responsible for the spatial dependence of $\overleftrightarrow{\epsilon}$. In the simplest approximation, spherical particles interacting with their own interface images were considered, ${ }^{6,18}$ leading to the expression

$$
\begin{aligned}
\epsilon_{x}= & \epsilon_{m}\left(1+\frac{3\left(\epsilon_{p}-\epsilon_{m}\right)}{\epsilon_{p}+2 \epsilon_{m}} f\right)-\frac{3 c_{x}}{8} f \epsilon_{m}^{\prime} \frac{\epsilon_{m}-\epsilon_{m}^{\prime}}{\epsilon_{m}+\epsilon_{m}^{\prime}} \frac{\left(\epsilon_{p}-\epsilon_{m}\right)^{2}}{\left(\epsilon_{p}+2 \epsilon_{m}\right)^{2}} \frac{\bar{a}^{3}}{z^{3}} \\
& +\cdots
\end{aligned}
$$

for the components of $\overleftrightarrow{\epsilon}$ written as $\overleftrightarrow{\epsilon}=\epsilon_{\mid} \vec{n} \vec{n}+\epsilon_{\perp}(\overleftrightarrow{I}-\vec{n} \vec{n})$. Here $x=\|, \perp\left(c_{\|}=2, c_{\perp}=1\right)$ denotes the longitudinal and transverse components of $\overleftrightarrow{\epsilon}, \vec{n}$ is a unit vector perpendicular to the interface, as shown in Fig. 1, and $\vec{I}$ is a unit $3 \times 3$ tensor. It is also assumed that the system consists of randomly dispersed spherical particles $\left(\epsilon_{p}\right)$ of mean radius $\bar{a}$ with the filling factor $f$, surrounded by a matrix material $\left(\epsilon_{m}\right)$. The dispersion adjoins a homogeneous medium $\left(\epsilon_{m}^{\prime}\right)$ in the right-hand side in Fig. 1.

One of the shortcomings of the approximation (1) is its restricted region of validity. In particular, the condition $\epsilon_{m}^{\prime}$ $\neq \epsilon_{m}$ must be satisfied. Otherwise the effect of electrostatic

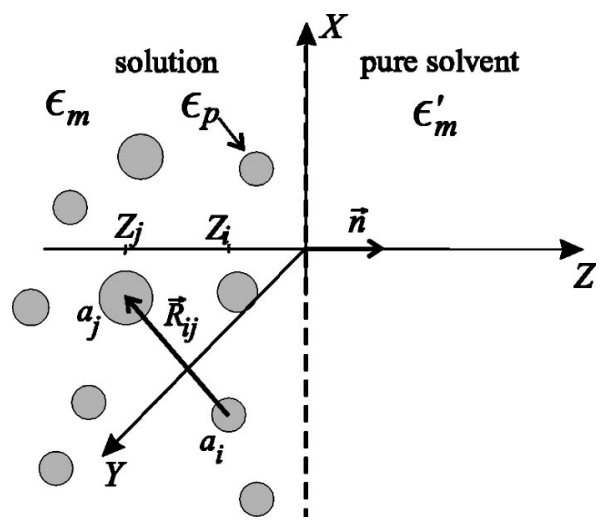

FIG. 1. Colloidal solution in the vicinity of an interface with pure solvent. If the opposite is not stated, $\epsilon_{m}^{\prime} \equiv \epsilon_{m}$ is assumed all throughout the paper. 
images will be negligible and Eq. (1) will not contain any spatial dependence. On the other hand, the liquid-liquid phase boundary between a colloidal solution and a pure solvent represents a typical example of the situation when $\epsilon_{m}^{\prime}$ $\equiv \epsilon_{m}$. Apparently, the $z$ dependence of the many-particle correlation function is responsible for the difference between $\epsilon$ in the interface layer and in the bulk. This also implies that the first nonvanishing $z$-dependent term in an Eq. (1)-like series will be caused by a particle-particle interaction and, thus, is proportional to $f^{2}$.

It is the purpose of this paper to obtain the effective dielectric function of a colloidal system in the vicinity of an interface with pure solvent. A technique developed earlier ${ }^{19}$ for accounting for two-particle interaction effects will be employed to obtain an explicit expression for $\overleftrightarrow{\epsilon}(z)$. Polydispersity corrections will be considered as well.

As has been shown in Ref. 18, the components of $\overleftrightarrow{\epsilon}$ can be determined via the polarizability tensor $\overleftrightarrow{A}(z)\left(\overleftrightarrow{A}\right.$ is defined ${ }^{20}$ as the proportionality coefficient between the dielectric polarization vector $\vec{P}$ and the external field $\vec{E}_{0}: \vec{P}=\overleftrightarrow{A} \vec{E}_{0}$ ) using the equations

$$
\begin{gathered}
\left(\frac{\epsilon_{\perp}}{\epsilon_{m}}-1\right)\left(1-\frac{4 \pi}{3} f_{1}\right)=4 \pi A_{\perp}, \\
\left(\frac{\epsilon_{\|}}{\epsilon_{m}}-1\right)\left(1-\frac{4 \pi}{3}\left(f_{1}+f_{2}\right)\right)=4 \pi A_{\|},
\end{gathered}
$$

where $f_{1}$ and $f_{2}$ are functions of $A_{\perp, \|}$ and their explicit form is given by Eq. 6 from Ref. 18. In turn, the polarizability tensor $\overleftrightarrow{A}$ of the colloidal system is related ${ }^{21}$ to the set of irreducible polarizabilities $\vec{\alpha}^{(k)}$ of groups of $k$ particles

$$
\overleftrightarrow{A}=\frac{1}{V}\left(N\left\langle\overleftrightarrow{\alpha}^{(1)}\right\rangle+\frac{N(N-1)}{2}\left\langle\overleftrightarrow{\alpha}^{(2)}\right\rangle+\cdots\right) .
$$

Here $N$ and $V$ are the number of particles and the volume of the system, respectively, and $\langle\cdots\rangle$ denotes the average over the shape, size, and positions of the inclusions. In the $\epsilon_{m}$ $\neq \epsilon_{m}^{\prime}$ case, $\overleftrightarrow{\alpha}^{(1)}$ is the first nontrivial $z$-dependent term and is caused by the interaction between the particle and its electrostatic image.$^{18}$ However, for the situation addressed here, where a solution is in equilibrium with a pure solvent, $\epsilon_{m}$ $\equiv \epsilon_{m}^{\prime}$, and therefore no electrostatic images are present. Let us consider spherical particles with continuously distributed radii $(i, j=1, \ldots, N$ are used further on to number the particles). The polarizability of a single sphere of radius $a_{i}$ is the same as in the absence of the interface and reads

$$
\stackrel{\leftrightarrow}{\alpha}^{(1)}(i)=-a_{i}^{3} \kappa \stackrel{\leftrightarrow}{I}
$$

with $\kappa=\left(\epsilon_{m}-\epsilon_{p}\right) /\left(2 \epsilon_{m}+\epsilon_{p}\right)$. The coefficients $f_{1,2}$, calculated taking into account only one-particle effects, are also trivial:

$$
f_{1}=-\frac{3}{4 \pi} \kappa f \quad \text { and } \quad f_{2}=0 .
$$

Thus, as expected, the set of equations (2) in the linear-upon$f$ approximation leads to $\epsilon_{\perp}=\epsilon_{\|}=\epsilon_{m}(1-3 \kappa f)$, the linearized

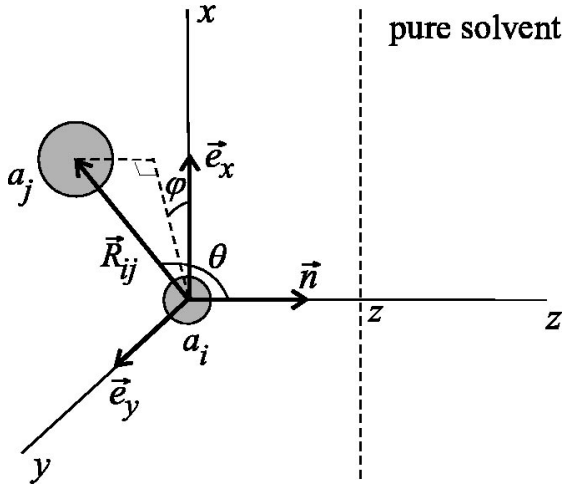

FIG. 2. A pair of particles in the vicinity of the phase boundary.

Maxwell-Garnett ${ }^{2}$ relation, and includes no spatial dependence [the identity $f \equiv(4 \pi / 3)\left\langle a_{i}^{3}\right\rangle N / V$ has been used].

Hence, to take into account the spatial dependence of the dielectric function $\overleftrightarrow{\epsilon}$ in the case of a solution-solvent system, one has to consider the higher order terms in Eq. (3), the first of which is expressed by $\left\langle\leftrightarrow^{(2)}\right\rangle$. The physical origin of the $\left\langle\overleftrightarrow{\alpha}^{(2)}\right\rangle$ spatial dependence is quite clear: the pair correlation function $g_{2}$ depends on the distances $Z_{i, j}$ from the interface. In other words, a particle in the vicinity of the boundary, Fig. 1 , has many more neighbors on the left-hand side than to the right, whereas a particle in the bulk finds itself in an "almost isotropic" environment. In general, the correlation function $g_{2}$ can be borrowed from the theory of fluids. ${ }^{22}$ However, even the simplest approximation, reflecting the asymmetry in the two-particle distribution function, e.g.,

$g_{2}\left(\vec{r}_{i}, \vec{r}_{j}\right)= \begin{cases}0, & R_{i j}<a_{i}+a_{j} \text { or } Z_{i}>0 \text { or } Z_{j}>0, \\ 1 / V^{2} & \text { otherwise },\end{cases}$

is enough for our purpose. Here $\vec{r}_{i}=\left(X_{i}, Y_{i}, Z_{i}\right)$ describes the position of the particle $i$ and $R_{i j}=\left|\vec{r}_{j}-\vec{r}_{i}\right|$ (Fig. 1). In the approximation (6) the particles are assumed to be hard spheres (of radii $a_{i}$ and $a_{j}$ ) uniformly distributed in the matrix. More conveniently, the pair distribution function can be written in the local system of coordinates with the origin at the center of the particle $i$, as shown in Fig. 2:

$$
g\left(\vec{R}_{i j}\right)= \begin{cases}0 & R_{i j}<a_{i}+a_{j} \text { or }\left(\vec{R}_{i j} \vec{n}\right)>z, \\ 1 / V & \text { otherwise }\end{cases}
$$

Here $z>0$ and the function $g\left(\overleftrightarrow{R}_{i j}\right)$ vanishes if vector $\vec{R}_{i j}$ points outside the solution volume, i.e., if $\left(\vec{R}_{i j} \vec{n}\right)>z$. The function $g(\vec{R})$ gives the probability density to find a particle at the distance $\vec{R}$ from the given one if the latter is located at distance $z$ from the interface. This function should be used to average over $R_{i j}$ in the expressions for polarizability (see below).

In turn, the two-particle polarizability can be determined from the relation $\vec{d}^{(2)}=\alpha^{(2)} \vec{E}_{0}$, where $\vec{d}^{(2)}$ is the dipole moment gained by the pair due to the mutual polarization of the particles $i$ and $j$. Due to the symmetry, it has only two independent components and can be represented as 


$$
\stackrel{\leftrightarrow}{\alpha}^{(2)}(i, j)=\alpha_{l}\left(R_{i j}\right) \vec{e}_{R} \vec{e}_{R}+\alpha_{t}\left(R_{i j}\right)\left(\stackrel{\leftrightarrow}{I}-\vec{e}_{R} \vec{e}_{R}\right)
$$

where $\vec{e}_{R}=\vec{R}_{i j} / R_{i j}$ (see Fig. 2), and $\alpha_{l}$ and $\alpha_{t}$ are scalar polarizabilities of the pair in longitudinal $\left(\vec{E}_{0} \| \vec{R}\right)$ and transverse $\left(\vec{E}_{0} \perp \vec{R}\right)$ fields, which are to be calculated independently. ${ }^{19,21}$
Using the distribution function (7) and the representation (8), the averaging over positions of the particles can be performed in Eq. (3). The orientation of a pair is described by the unit vector $\vec{e}_{R}(\theta, \varphi)=\cos \theta \vec{n}+\sin \theta \cos \varphi \vec{e}_{x}$ $+\sin \theta \sin \varphi \vec{e}_{y}$ (Fig. 2) and the averaging over the angles $\theta$ and $\varphi$ is conducted explicitly:

$$
\begin{gathered}
A_{\|}=A_{b u l k}-\frac{\pi}{3} c^{2}\left\langle\int_{z}^{\infty} R_{i j}^{2} d R_{i j}\left(\alpha_{l}\left(R_{i j}\right)+2 \alpha_{t}\left(R_{i j}\right)-3 \alpha_{t}\left(R_{i j}\right) \frac{z}{R_{i j}}+\left[\alpha_{t}\left(R_{i j}\right)-\alpha_{l}\left(R_{i j}\right)\right] \frac{z^{3}}{R_{i} j^{3}}\right)\right\rangle_{a}, \\
A_{\perp}=A_{\text {bulk }}-\frac{\pi}{3} c^{2}\left\langle\int_{z}^{\infty} R_{i j}^{2} d R_{i j}\left(\alpha_{l}\left(R_{i j}\right)+2 \alpha_{t}\left(R_{i j}\right)-\frac{3}{2}\left[\alpha_{t}\left(R_{i j}\right)+\alpha_{l}\left(R_{i j}\right)\right] \frac{z}{R_{i j}}-\frac{1}{2}\left[\alpha_{t}\left(R_{i j}\right)-\alpha_{l}\left(R_{i j}\right)\right] \frac{z^{3}}{R_{i j}^{3}}\right)\right\rangle_{a},
\end{gathered}
$$

where $c=N / V$ is the density of inclusions, $\langle\cdots\rangle_{a}$ denotes the average over the inclusion sizes $a_{i, j}$, and

$$
A_{\text {bulk }}=-\frac{3 f \kappa}{4 \pi}+\frac{2 \pi}{3} c^{2}\left\langle\int_{a+b}^{\infty} R_{i j}^{2} d R_{i j}\left[\alpha_{l}\left(R_{i j}\right)+2 \alpha_{t}\left(R_{i j}\right)\right]\right\rangle_{a}
$$

is the polarizability in the bulk of the colloidal solution. Note, the expression (9) is an expansion in powers of $c$ truncated at the two-particle $c^{2}$ term.

To proceed, the scalar two-particle polarizabilities have to be calculated. In particular, for spherical particles this can be done by the method presented in Ref. 19. Here we only briefly outline the approach. Its essence is in the iterative procedure where the repolarization operator $\hat{\mathcal{P}}(j, i)$ can be introduced in such a way that at each iteration step $s$ the multipole moments $G_{k}^{(s)}(j)$ of the particle $j$ induced by the particle $i$ are written as

$$
G_{k}^{(s)}(j)=G_{k}^{(s-1)}(j)+\sum_{n} \mathcal{P}_{k n}(j, i) G_{n}^{(s-1)}(i),
$$

where $\left\{G_{n}(i)\right\}$ is the set of multipole moments of the particle $i$. For example, in the case of the longitudinal field $\vec{E}_{0}$, corresponding to $\alpha_{l}$, the operator $\hat{\mathcal{P}}$ has the form ${ }^{19}$

$$
\mathcal{P}_{k n}(j, i)=\frac{(-1)^{n}(n+k) !}{n ! k !} \sqrt{\frac{2 n+1}{2 k+1}} \frac{a_{j}^{2 k+1}}{R_{i j}^{n+k+1}} \kappa(k)
$$

with $\kappa(k)=\left(\epsilon_{m}-\epsilon_{p}\right) k /\left[\epsilon_{m}(k+1)+\epsilon_{p} k\right]$. Together with the relation

$$
G_{k}^{(s)}(i)=G_{k}^{(s-1)}(i)+\sum_{n} \mathcal{P}_{k n}(i, j) G_{n}^{(s-1)}(j)
$$

we obtain a closed set of equations. To apply the iterative scheme, one also needs the initial multipole moments, i.e., the multipole moments induced by the polarization of noninteracting particles in the field $\vec{E}_{0}: G_{1}^{(0)}(i)=-2 \sqrt{\pi / 3} a_{i}^{3} E_{0} \kappa$ and $G_{k}^{(0)}(i)=0, k>1, i=1, \ldots, N$. The polarizabilities $\alpha_{l}$ and $\alpha_{t}$, written with an arbitrary precision as a series upon $a_{i} / R_{i j}$ and $a_{j} / R_{i j}$ parameters, are the outcome of the approach. ${ }^{19}$

To avoid bulky formulas, we shall use them in a simplified form, retaining only the first two terms:

$$
\begin{aligned}
& \alpha_{l}\left(R_{i j}, a_{i}, a_{j}\right)=\frac{4 \kappa^{2} a_{i}^{3} a_{j}^{3}}{R_{i j}^{3}}-\frac{4 \kappa^{3}\left(a_{i}^{6} a_{j}^{3}+a_{j}^{6} a_{i}^{3}\right)}{R_{i j}^{6}}+\cdots, \\
& \alpha_{t}\left(R_{i j}, a_{i}, a_{j}\right)=-\frac{2 \kappa^{2} a_{i}^{3} a_{j}^{3}}{R_{i j}^{3}}-\frac{\kappa^{3}\left(a_{i}^{6} a_{j}^{3}+a_{j}^{6} a_{i}^{3}\right)}{R_{i j}^{6}}+\cdots .
\end{aligned}
$$

Expressions (13) are to be substituted into (9). The averages over the size of inclusions can be computed approximately assuming that the mean radius is $\bar{a}$, and the standard deviation $\Delta=\sqrt{\left\langle\left(a_{i}-\bar{a}\right)^{2}\right\rangle}$ is small compared to the mean radius. ${ }^{23}$ To reach accuracy up to $f^{2}$ (two-particle effects) it is enough to use Eq. (5) for $f_{1,2}$ to calculate $\epsilon_{\perp}$ and $\epsilon_{\|}$in Eq. (2). Hence, the final result reads

$$
\begin{aligned}
& \frac{\epsilon_{\perp}-\epsilon_{\text {bulk }}}{\epsilon_{m}}=\frac{9}{16}\left(\frac{\epsilon_{m}-\epsilon_{p}}{2 \epsilon_{m}+\epsilon_{p}}\right)^{3}\left(1+12 \frac{\Delta^{2}}{\bar{a}^{2}}\right) f^{2} \frac{\bar{a}^{3}}{z^{3}}, \\
& \frac{\epsilon_{\|}-\epsilon_{\text {bulk }}}{\epsilon_{m}}=\frac{9}{32}\left(\frac{\epsilon_{m}-\epsilon_{p}}{2 \epsilon_{m}+\epsilon_{p}}\right)^{3}\left(1+12 \frac{\Delta^{2}}{\bar{a}^{2}}\right) f^{2} \frac{\bar{a}^{3}}{z^{3}},
\end{aligned}
$$

where $\epsilon_{b u l k}$ is the dielectric function of the solution far from the interface. $\epsilon_{\text {bulk }}$ has been calculated in Ref. 19 as a power series of $\kappa$; i.e., its leading terms can be written in the form $^{24}$

$$
\frac{\epsilon_{b u l k}-\epsilon_{m}}{\epsilon_{m}}=-3 f \kappa\left[1-\kappa f+\kappa^{2} f\left(0.421+0.237 \frac{\Delta^{2}}{\bar{a}^{2}}\right)\right],
$$

which agrees with results found in the literature. ${ }^{9}$

We retained the terms up to $1 / R_{i j}^{6}$ in the polarizabilities (13). Consequently, only the first nonvanishing term, proportional to $1 / z^{3}$, is present in Eq. (14). This, in particular, implies the restriction $\bar{a}<z$, which is assumed to be fulfilled. A more serious limitation of Eq. (14) is the fact that only pair 
interactions were taken into account. It makes the result applicable for the case of $f \ll 1$ only, where three- and moreparticle effects can be neglected.

It is interesting that the influence of polydispersity is well pronounced and cannot be neglected. Indeed, the prefactor 12 in Eq. (14) suggests the importance of the size distribution effect. This also supports the point observed in bulk systems: $:^{5,13}$ the effective permittivity depends in a strong way on the polydispersity of the inclusions.

While deriving Eq. (14) we completely neglected the fact that $f$ is a function of $z$ near the interface. Indeed, one expects the filling fraction $\varphi(z)$ to be a smoothed steplike function described by some characteristic "interface width" $\xi(\xi>\bar{a})$, such that $\varphi(z \gg \xi)=f$ and $\varphi(z<0)=0$. An accurate incorporation of such a space-dependent filling factor considerably complicates the theory. However, if $\xi>\bar{a}$, a rough approximation can be made substituting $\varphi(z)$ instead of $f$ in Eqs. (14) and (15). Let us briefly discuss some possible consequences of such an ansatz for the analysis of reflectance spectra. Generally the refractive index profile in the vicinity of an interface is modeled by a weighted sum of the refractive indices of the phases $n_{1}$ and $n_{2}$. In our case $n_{1}$ corresponds to the solution refractive index in the bulk and $n_{2}$ to the one of the solvent. Both are constant for a fixed $f$, i.e., they do not depend on $z$. This yields ${ }^{17} n(z)=n_{1} \varphi(z) / f+n_{2}[1$ $-\varphi(z) / f]$. However, in the case $\xi>\lambda$, where $\lambda$ is the wavelength of the incident and reflected light, the $z$ dependence of the refractive index of the disordered medium $n_{1}$ might become important. Indeed, if $\lambda$ is chosen in such a way that $\lambda<\xi$ and $\lambda>\bar{a}$ (the latter inequality ensures the dielectric function can be properly defined), the expansion (14) with $\varphi(z)$ instead of $f$ can be used. Analysis of this case would allow one to determine the density profile on the scale of $\xi$ if $\bar{a}$ is known, or vice versa. It has to be admitted, however, that at the moment we cannot give an example, available in the literature, where the picture presented above can be worked out. Still, we believe that this analysis could be potentially interesting.

Throughout the paper we considered a system consisting of a colloidal solution in equilibrium with a pure solvent $\left(\epsilon_{m} \equiv \epsilon_{m}^{\prime}\right.$, Fig. 1). However, the same kind of calculation can be used to account for the interface effects in the vicinity of a boundary between a microinhomogeneous medium and a homogeneous dielectric. If the material of the dielectric is not the same as the matrix of the composite, $\epsilon_{m} \neq \epsilon_{m}^{\prime}$, the influence of electrostatic images should be also taken into account. ${ }^{18}$ Apparently, the smaller the difference $\left|\epsilon_{m}-\epsilon_{m}^{\prime}\right|$, the more important the anisotropic two-particle effects are if compared to the contribution from electrostatic one-particle images. A simple estimate follows from Eqs. (1) and (14), indicating the region where Eq. (14) holds:

$$
\left|\frac{\epsilon_{m}-\epsilon_{m}^{\prime}}{\epsilon_{m}+\epsilon_{m}^{\prime}}\right|<\left(\frac{\epsilon_{m}-\epsilon_{p}}{2 \epsilon_{m}+\epsilon_{p}}\right)^{2} f \text {. }
$$

Outside this region both effects are to be incorporated into the model. Although possible, this would lead to much bulkier formulas.

The author thanks Professor N. P. Malomuzh for introducing him to this field, and for many fruitful discussions.
*Electronic address: r.stepanyan@tn.utwente.nl

${ }^{1}$ J. C. Maxwell, A Treatise on Electricity and Magnetism, 2nd ed. (Dover, New York, 1954), reprinted from the 3rd ed., Clarendon, Oxford, 1891.

${ }^{2}$ J. C. M. Garnett, Philos. Trans. R. Soc. London, Ser. A 203, 385 (1904).

${ }^{3}$ K. W. Wagner, Arch. Electrochem. 2, 371 (1914).

${ }^{4}$ B. U. Felderhof and R. B. Jones, Phys. Rev. B 39, 5669 (1989).

${ }^{5}$ R. G. Barrera, P. Villaseñor-González, W. L. Mochán, and G. Monsivais, Phys. Rev. B 41, 7370 (1990).

${ }^{6}$ S. V. Kuzmin and N. P. Malomuzh, J. Mol. Liq. 58, 81 (1993).

${ }^{7}$ L. Fu, P. B. Macedo, and L. Resca, Phys. Rev. B 47, 13818 (1993).

${ }^{8}$ S. E. Skipetrov, Phys. Rev. B 60, 12705 (1999).

${ }^{9}$ K. Hinsen and B. U. Felderhof, Phys. Rev. B 46, 12955 (1992).

${ }^{10}$ R. Pelster, Phys. Rev. B 59, 9214 (1999).

${ }^{11}$ R. Pelster and U. Simon, Colloid Polym. Sci. 277, 2 (1999).

${ }^{12}$ K. Günter and D. Heinrich, Z. Phys. 185, 345 (1965).

${ }^{13}$ A. Spanoudaki and R. Pelster, Phys. Rev. B 64, 064205 (2001).

${ }^{14}$ R. G. Barrera, M. del Castillo-Mussot, G. Monsivais, P. Villaseñor, and W. L. Mochán, Phys. Rev. B 43, 13819 (1991).

${ }^{15}$ J. Vlieger and D. Bedeaux, Thin Solid Films 69, 107 (1980); D. Bedeaux and J. Vlieger, ibid. 102, 265 (1983).

${ }^{16}$ M. M. Wind, J. Vlieger, and D. Bedeaux, Physica A 141, 33 (1987); P. A. Bobbert and J. Vlieger, ibid. 147, 115 (1987); M.
M. Wind, P. A. Bobbert, J. Vlieger, and D. Bedeaux, ibid. 143, 164 (1987).

${ }^{17}$ J. Schulz, A. Hirtz, and G. H. Findenegg, Physica A 244, 334 (1997); G. H. Findenegg, A. Hirtz, and R. Subman, in Abstracts of 4th European Colloid \& Interface Society Conference, Catanzaro, Italy, 1990; E. H. Hoog, H. N. Lekkerkerker, J. Schulz, and G. H. Findenegg, J. Phys. Chem. B 103, 10657 (1999); A. Hirtz, W. Lawnik, and G. H. Findenegg, Colloids Surf. 51, 405 (1990).

${ }^{18}$ R. Stepanyan, Phys. Rev. B 67, 073403 (2003).

${ }^{19}$ R. Stepanyan, Russian Colloid Journal 61, 781 (1999).

${ }^{20}$ J. D. Jackson, Classical Electrodynamics (Wiley, New York, 1975).

${ }^{21}$ T. Lokotosh and N. Malomuzh, Russian J. of Physical Chemistry 68, 885 (1994).

${ }^{22}$ I. Z. Fisher, The Statistical Theory of Liquids (University of Chicago Press, Chicago, 1964).

${ }^{23}$ This corresponds to a continuous size distribution described by the Guassian $p(a)=\exp \left[-(a-\bar{a})^{2} /\left(2 \Delta^{2}\right)\right] /(\sqrt{2 \pi} \Delta)$ with small dispersion. The averages are computed as $\left\langle h\left(a_{i}, a_{j}\right)\right\rangle$ $=\int_{0}^{\infty} d a_{i} \int_{0}^{\infty} d a_{j} p\left(a_{j}\right) h\left(a_{i}, a_{j}\right)$.

${ }^{24}$ For this purpose one needs more terms in the expansion of $\alpha(\mathrm{R})$. The full series can be calculated for arbitrary $\epsilon_{m}$ and $\epsilon_{p}$. Then using Eq. (10) and a (2)-like equation for the bulk, one obtains $\epsilon_{b u l k}$. For the interfacial terms, if we restrict ourselves to the first $\bar{a} / z$ contribution, the truncated expansions (13) are sufficient. 